Correlation of Laser-Induced Damage to Phase Objects in Bulk Fused Silica

M. C. Nostrand, C. J. Cerjan, M. A. Johnson, T. I. Suratwala, T. L. Weiland, W. D. Sell, J. L. Vickers, R. L. Luthi, J. R. Stanley, T. G. Parham, C. B. Thorsness

November 11, 2004

Boulder Damage Symposium XXXVI Annual Symposium on Boulder, CO, United States September 20, 2004 through September 22, 2004 
This document was prepared as an account of work sponsored by an agency of the United States Government. Neither the United States Government nor the University of California nor any of their employees, makes any warranty, express or implied, or assumes any legal liability or responsibility for the accuracy, completeness, or usefulness of any information, apparatus, product, or process disclosed, or represents that its use would not infringe privately owned rights. Reference herein to any specific commercial product, process, or service by trade name, trademark, manufacturer, or otherwise, does not necessarily constitute or imply its endorsement, recommendation, or favoring by the United States Government or the University of California. The views and opinions of authors expressed herein do not necessarily state or reflect those of the United States Government or the University of California, and shall not be used for advertising or product endorsement purposes. 


\title{
Correlation of Laser-induced Damage to Phase Objects in Bulk Fused Silica
}

\author{
M.C. Nostrand*, C. J. Cerjan, M. A. Johnson, T. I. Suratwala, T. L. Weiland, W. D. Sell, \\ J. L. Vickers, R. L. Luthi, J. R. Stanley, T. G. Parham, C. B. Thorsness \\ Lawrence Livermore National Laboratory \\ 7000 East Avenue, L-592 \\ Livermore, CA 94550
}

\begin{abstract}
The Optical Sciences Laser (OSL) Upgrade facility, described in last year's proceedings ${ }^{1}$, is a kJ-class, large aperture $\left(100 \mathrm{~cm}^{2}\right)$ laser system that can accommodate prototype optical components for large-scale inertial confinement fusion lasers. High-energy operation of such lasers is often limited by damage to the optical components. Recent experiments on the OSL Upgrade facility using fused silica components at $4 \mathrm{~J} / \mathrm{cm}^{2}(351-$ $\mathrm{nm}$, 3-ns) have created output surface and bulk damage sites that have been correlated to phase objects in the bulk of the material. Optical Path Difference (OPD) measurements of the phase defects indicate the probability of laser-induced damage is strongly dependent on OPD.
\end{abstract}

\section{INTRODUCTION}

Laser-induced damage to optical components is a limitation of high-fluence laser systems such as the National Ignition Facility (NIF), particularly at UV-wavelengths near $350 \mathrm{~nm}$. Previous experiments at LLNL have revealed a bulk defect in Corning 7980 fused silica responsible for damage at relatively low average 350-nm fluences of 2-4 J/cm ${ }^{2}(3 \mathrm{~ns})^{2}$. These defects are localized index inhomogeneities that locally focus the laser beam to a much higher fluence, thereby causing output surface damage, see Figure 1 . These index inhomogeneities behave like little lenses, or "lenslets", and thus are pure phase objects which scatter light predominantly in the forward direction. Accordingly, these lenslets only become visible when they are backlit by a uniform light source and viewed out of the plane of the object, see Figure 2. These objects are typically on the order of $150-\mu \mathrm{m}$ in diameter, so non-destructively locating them on a $40-\mathrm{cm}$ optic can be challenging. As shown in Figure 3, lenslet identification after a damaging laser pulse is quite straightforward with proper lighting. While previous experience with lenslets was limited to post-damage identification, this study aims to non-destructively characterize the lenslet content of $40-\mathrm{cm}$ optics before laser exposure, and then correlate lenslet-induced laser damage to measured lenslet properties.

It should be noted that Corning 7980 fused silica optics are being used throughout the 1- $\mu$ m section of NIF. In particular, lenslet-induced damage probability is much less for the $1-\mu \mathrm{m}$ optics. Additionally, recent improvements in the glass manufacturing process for 7980 have reduced its lenslet content.

Details of the operation and configuration of the OSL Upgrade laser, where these experiments were performed, are given in Ref. [1]. The optic under test in these experiments is a wedged focus lens, located behind the tripling crystal.

\footnotetext{
* Correspondence: 925 422-2712, nostrand1@llnl.gov
} 


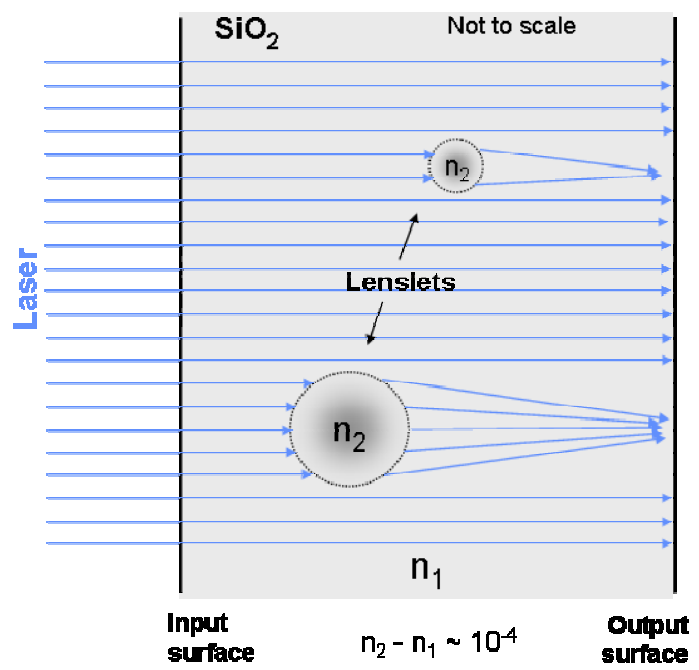

Figure 1. A schematic of the effect of index inhomogeneities on the input laser beam. These defects act like "lenslets" by focusing the incoming laser light towards the output surface.

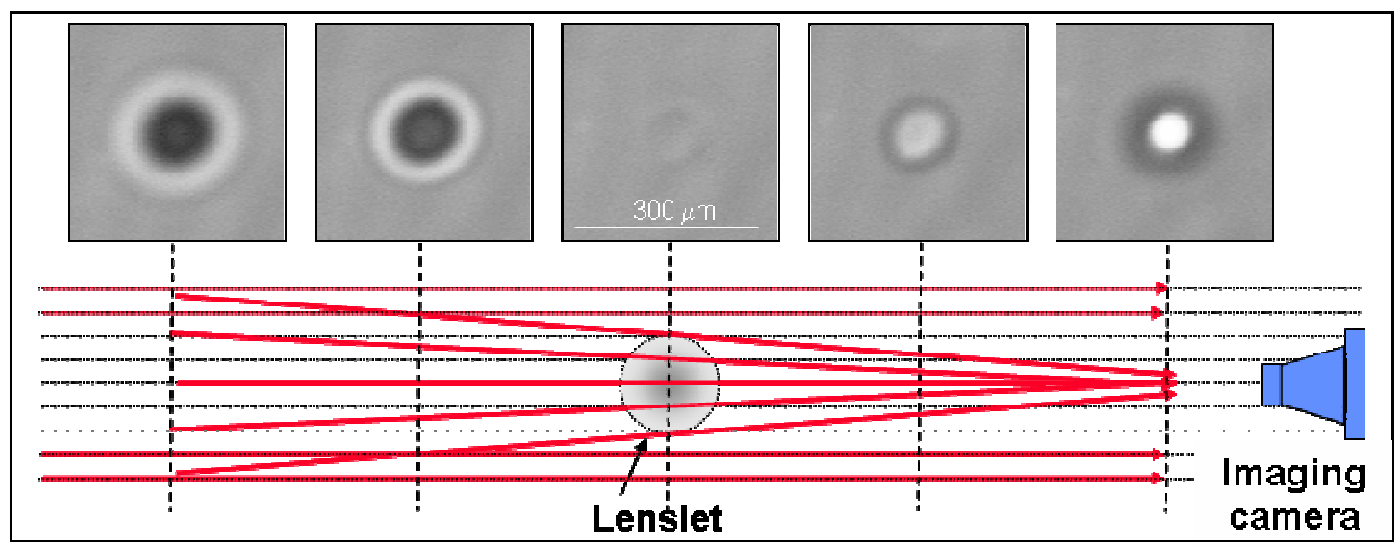

Figure 2. Lenslets are pure phase objects which scatter predominantly in the forward direction, and "disappear” when imaged in-plane. The horizontal dashed lines represent incident rays in the absence of the lenslet (background), while the solid arrows represent the apparent rays as viewed from the imaging camera. The vertical dashed lines represent different image planes along the z-axis.

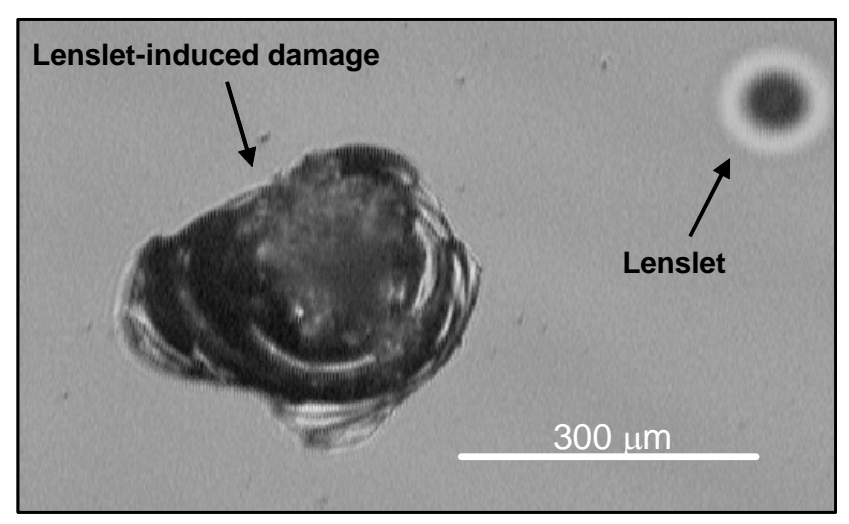

Figure 3. Identification of a lenslet after it has led to laser-induced damage is straightforward with proper backlit imaging. This image of output surface damage was taken looking through the input surface, thus the lenslet is between the damage site and camera in this case. 


\section{PRE-CHARACTERIZATION OF THE PHASE DEFECTS}

In order to non-destructively characterize the lenslet content of the test optic, a backlit mapping station was constructed in another facility. Ideally, a low-power $3 \omega$ laser source would be used for backlighting, so that the transmitted light could be monitored downstream for intensification. In practice, however, noise due to laser speckle prohibited the detection of small features on large optics in a reasonable amount of time. Thus an incoherent halogen source was used along with a narrow (10-nm) bandpass filter. 550-nm green light was used primarily for convenience. Highly uniform illumination was achieved by allowing the filtered source light to diverge greatly so that only the axial rays were used to illuminate the sample. A schematic of the set-up is shown in Figure 4. The key optical components are a light source, a condensing lens, an imaging lens, and a CCD camera.

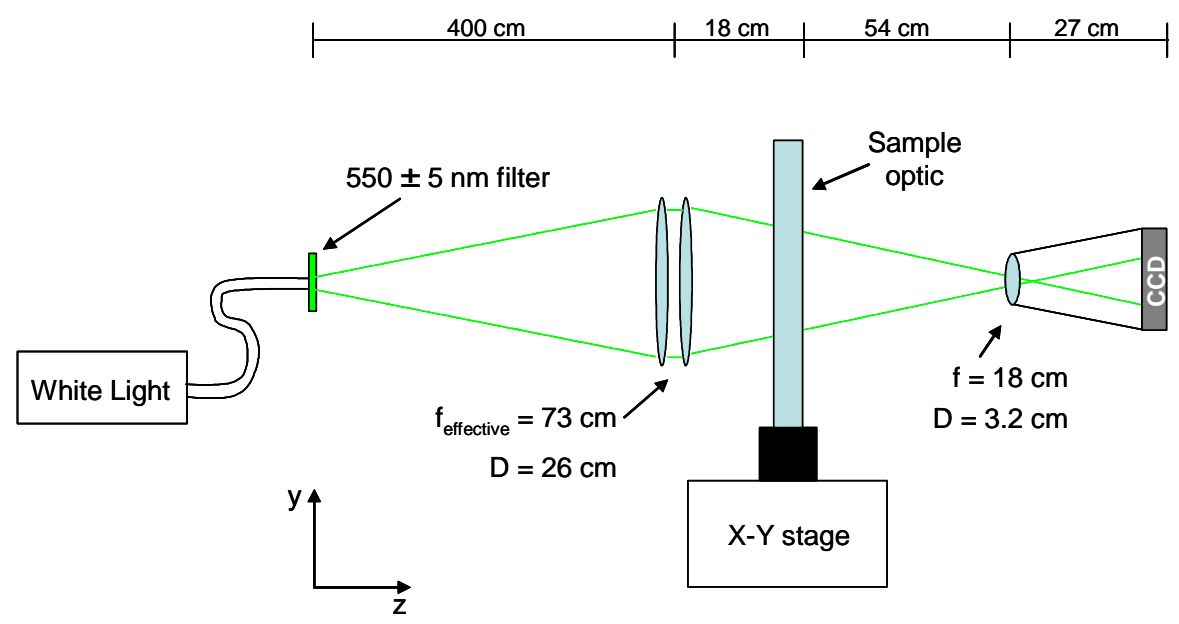

Figure 4. Schematic of backlit mapping set-up used to identify lenslets in 40-cm fused silica optics.

A large $x-y$ stage is used to move the sample. Since the system views the modulation from the features (and not the features themselves), it was determined that a resolution of $25 \mu \mathrm{m} /$ pixel was sufficient to identify and characterize the relevant features. Using lenses and the CCD camera available on-hand, each image can cover a 150-mm x 150$\mathrm{mm}$ patch of the sample, so that a $3 \times 3$ array of images is required to cover the $40-\mathrm{cm} \times 40-\mathrm{cm}$ optic. Each of the nine sub-regions will be referred to as a sector. Currently, the aperture-limiting component is the condensing lens. Optimized optical designs and state-of-the-art CCD cameras could increase the per-image aperture (as well as resolution).

Data is obtained at two separate image planes. This allows modelers to use phase-recovery algorithms to predict the intensification at any plane downstream, as well as locate the feature in the bulk of the material. Preferably, the two images planes would coincide with the optical surfaces of the sample, allowing easier identification of surface artifacts (dust, residue, etc.) which can be otherwise indistinguishable from real bulk lenslets. For this reason, it is important to have the surfaces free of dust and other surface artifacts.

In practice, the image plane nearest the CCD camera (the output surface of the optic relative to the light source) is established by imaging a small ruler placed below the optic in the same plane as the surface. An image is taken of this ruler to establish the resolution. The input surface plane is then established by moving the CCD camera on a rail toward the sample a distance $t / n$, where $t$ is the sample thickness and $n$ is the refractive index $(n=1.46$ is used for the 550-nm light). The condenser lens is also moved a concomitant distance.

The nine images for the output surface are taken first, the CCD camera and condenser lens are then moved, and the nine input surface images are acquired last. The CCD camera is a BetterLight ${ }^{\mathrm{TM}}$ model 6000, which is a $6000 \times 1$ pixel array (12- $\mu$ m pixels) that is line-scanned to produce a $6000 \times 8000$ array. Each 8-bit, grayscale image utilizes $6000 \times 6000$ pixels or $35.2 \mathrm{MB}$, so the total amount of raw data for each optic is almost $640 \mathrm{MB}$. The scan time for each image is 50 seconds. 
Because we are looking for very small lenslets $\left(\sim 1600 \mu \mathrm{m}^{2}\right)$ on a very large optic $\left(\sim 1600 \mathrm{~cm}^{2}\right)$, image analysis is required in order to extract relevant lenslet information. As described above, lenslet mapping data comes in the form of image pairs. One image is taken near the input surface of the optic, the other near the output surface, so that the lenslet is necessarily located between the two images. Pattern-recognition software (currently IPLab ${ }^{\circledR}$ ) spatially registers the images in each pair, locates the lenslets within the raw images, and cuts out a 50 x 50 pixel image from each set to form an image pair for each lenslet. These image pairs are then passed through phase-retrieval algorithms to calculate optical path difference (OPD), proximity to a surface (z), and spatial extent (x,y) of the lenslet. Calibration measurements have been made on a $15-\mathrm{cm}$ test piece with a phase-shifting interferometer to validate the phase-retrieval calculations.

It should be noted that the term lenslet will be used hereafter to mean any form of index-variation feature flagged by the pattern-recognition software, including pure lenslets (i.e. pure phase objects), inclusions (i.e. pure amplitude objects), and all combinations in between. Also, since the system uses a pattern-recognition algorithm, there will always be cases of "false positives" (features such as surface dust flagged as lenslets) and "false negatives" (lenslets in the part not identified by the software). Currently, the sensitivity of the system is set such that the latter should be rare (less than $1 \%$ ), while the former could be on the order of a $10 \%$ problem.

The phase-retrieval problem has been extensively studied for several decades. The well-known Gerchberg-Saxton (G-S) algorithm has been analyzed and used in many contexts. A slight extension of this algorithm was found to be suitable for defect characterization in this context. Amplitudes at two distances $\left(\mathrm{f}_{1}, \mathrm{f}_{2}\right)$ from an imaging camera are supplied. To determine the phases $\left(\phi_{1}, \phi_{2}\right)$ that reconstruct the complete fields $g=f * \exp (i \phi)$, we propagate to the pure phase plane (where the amplitude vanishes) and estimate the OPD of the scattering object by fitting the spatial phase distribution using the following steps:

- $\mathrm{g}_{1}=\mathrm{f}_{1} * \exp \left(\mathrm{i} \xi_{1}\right)$ where $\xi_{1}$ is an initial estimate of the phase at plane 1 ; propagate to plane 2 using split-step propagation.

- $\mathrm{g}_{2}=\mathrm{f}_{2} * \exp \left(\mathrm{i} \xi_{2}\right)$ replace modulus with measured modulus $\mathrm{f}_{2}$ at plane 2; propagate backwards to plane 1 .

- $\mathrm{g}_{3}=\mathrm{f}_{1} * \exp \left(\mathrm{i} \xi_{3}\right)$ replace modulus with measured modulus $\mathrm{f}_{1}$ at plane 1 ; continue until convergence.

An example of the result of this algorithm is shown below in Figure 5.
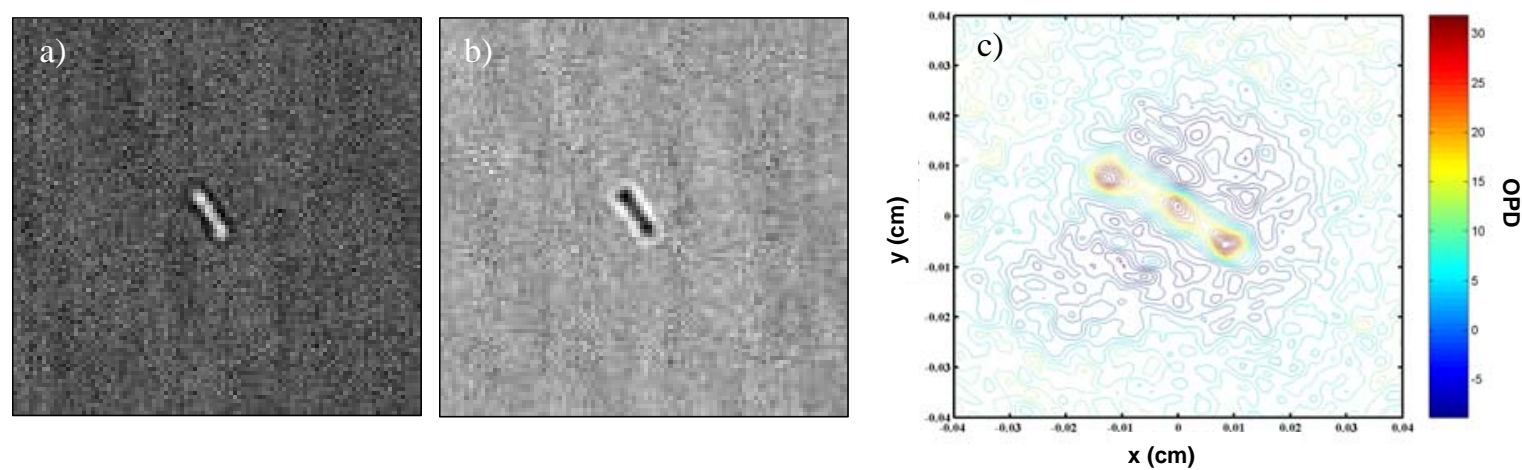

Figure 5. Example of phase-retrieval results (c) for input images (a) and (b).

The results of the pattern-recognition analysis are visually displayed as circles overlaid on a dark-field optic image. The circles locate the positions of lenslets in the sample. Lenslets are numbered according to their brightness (as found in the raw images). The results of the pattern-recognition analysis are shown in Figure 6. A total of 271 lenslets were identified. Examples of the raw mapping data for lenslets are also shown in the Figure. The clearaperture volume represents $\sim 4800 \mathrm{~cm}^{3}$ of material, so that the volume density of lenslets is $\sim 0.06 \mathrm{~cm}^{-3}$. 

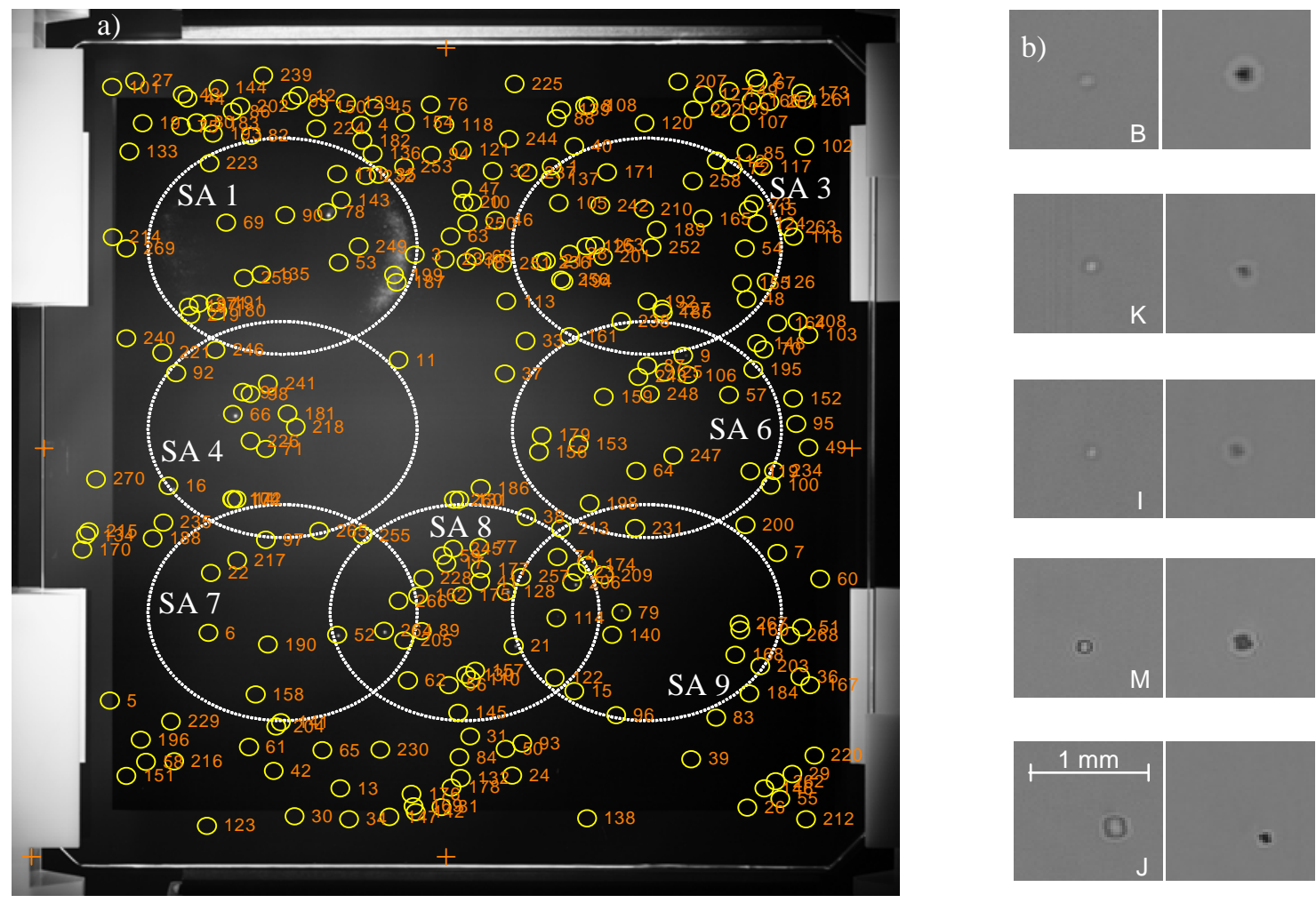

Figure 6. (a) Lenslet locations (circles) are overlaid on a side-lit photograph of the optic for visual display. (b) Examples of the raw data for some of the lenslets: output surface at left, corresponding input surface at right. Each of these lenslets led to output surface damage, the letter designation of the damage site is indicated. OSL Upgrade laser beam sub-apertures (SA, large ovals) are shown overlaid on the image.

Histograms of the results of the phase-retrieval analysis for the 271 lenslets are shown in Figure 7. Size of the lenslet is calculated as $\operatorname{sqrt}(\mathrm{x} \cdot \mathrm{y})$, where $\mathrm{x}$ is horizontal width and $\mathrm{y}$ the vertical width. Of the 271 lenslets in the entire part, only 116 fell within the aperture of the OSL Upgrade $4 \mathrm{~J} / \mathrm{cm}^{2}$ laser beams (see Figure 6). Results of the phase-retrieval analysis for these 116 lenslets are given in Table 1. The sub-aperture (SA) number of the OSL Upgrade beam that hit the lenslet is indicated in the table. Multiple entries indicate the lenslet was in a region of overlapping beams. The table is sorted by OPD.
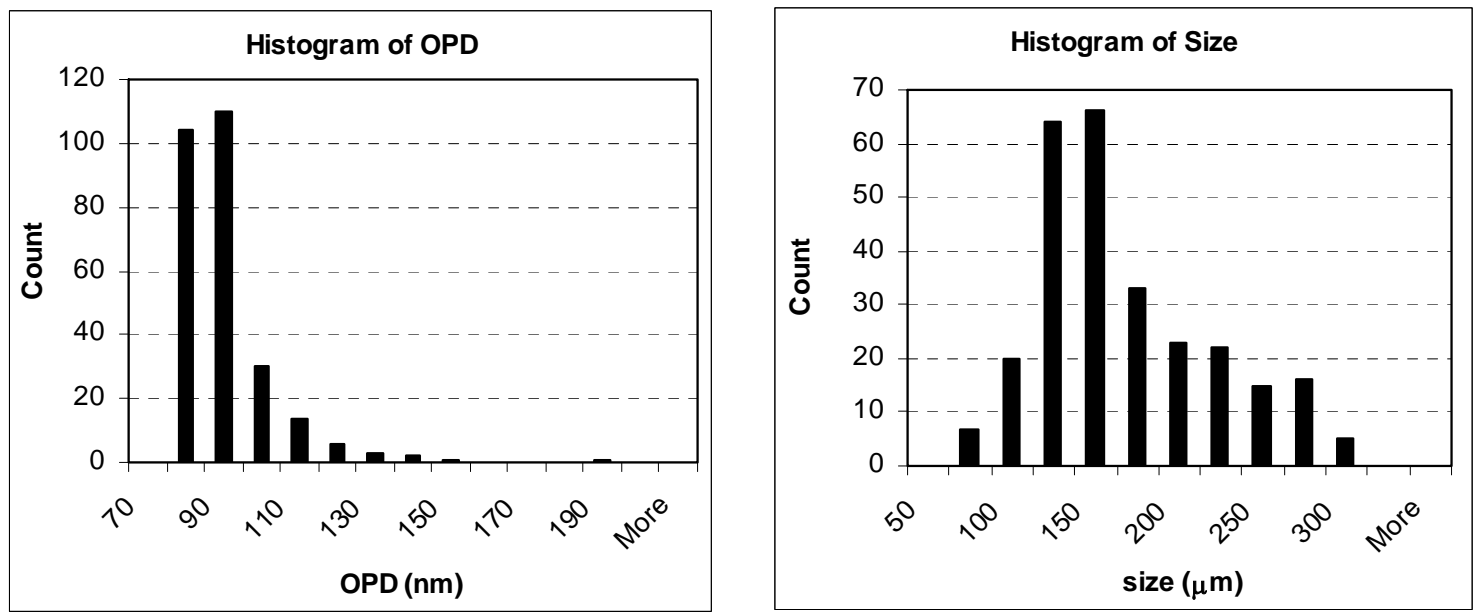

Figure 7. Histograms of the phase-retrieval analysis for the 271 lenslets identified in the test optic. 
Table 1. Results of phase-retrieval analysis on the 116 lenslets that fell within the OSL Upgrade $4 \mathrm{~J} / \mathrm{cm}^{2}$ clear-aperture. A letter designation in the last column indicates associated damage. The $x-y$ coordinates are relative to an origin at the lower left corner of the optic. The flat surface of the lens is located at $\mathrm{z}=0$.

\begin{tabular}{|c|c|c|c|c|c|c|c|c|c|c|c|c|c|c|c|c|c|}
\hline $\begin{array}{c}\text { Meas. } \\
\quad \#\end{array}$ & $\begin{array}{c}\mathbf{X} \\
(\mathrm{mm})\end{array}$ & $\begin{array}{c}\mathbf{y} \\
(\mathrm{mm})\end{array}$ & $\begin{array}{c}\mathrm{Z} \\
(\mathrm{mm})\end{array}$ & $\begin{array}{l}\text { size } \\
(\mu \mathrm{m})\end{array}$ & $\begin{array}{l}\text { OPD } \\
(\mathrm{nm})\end{array}$ & $\begin{array}{l}\text { OPD } \\
\text { rank }\end{array}$ & SA & Letter & $\begin{array}{c}\text { Meas. } \\
\#\end{array}$ & $\begin{array}{c}X \\
(\mathrm{~mm})\end{array}$ & $\begin{array}{c}y \\
(\mathrm{~mm})\end{array}$ & $\begin{array}{c}\mathrm{Z} \\
(\mathrm{mm})\end{array}$ & $\begin{array}{l}\text { size } \\
(\mu \mathrm{m})\end{array}$ & $\begin{array}{l}\text { OPD } \\
(\mathrm{nm})\end{array}$ & $\begin{array}{l}\text { OPD } \\
\text { rank }\end{array}$ & SA & Letter \\
\hline 25 & 332 & 253 & 6 & 285 & 180 & 1 & 6 & $\mathrm{~J}$ & 161 & 282 & 272 & 21 & 291 & 82 & 59 & 3 & \\
\hline 16 & 72 & 194 & 6 & 113 & 142 & 2 & 4 & $M$ & 157 & 233 & 97 & 20 & 292 & 82 & 60 & 8 & \\
\hline 23 & 286 & 149 & 6 & 249 & 139 & 3 & 9 & $\mathrm{H}$ & 111 & 160 & 357 & 20 & 315 & 82 & 61 & 1 & \\
\hline 66 & 106 & 231 & 19 & 216 & 131 & 4 & 4 & B & 191 & 96 & 290 & 32 & 241 & 82 & 62 & 1 & \\
\hline 52 & 160 & 116 & 19 & 195 & 126 & 5 & 7,8 & C & 187 & 191 & 300 & 32 & 267 & 82 & 63 & 1 & \\
\hline 6 & 93 & 117 & 6 & 200 & 113 & 6 & 7 & & 145 & 224 & 75 & 20 & 326 & 82 & 64 & 8 & \\
\hline 22 & 94 & 148 & 6 & 215 & 108 & 7 & 7 & & 115 & 376 & 339 & 20 & 333 & 81 & 65 & 3 & \\
\hline 17 & 218 & 153 & 6 & 217 & 105 & 8 & 8 & & 266 & 193 & 134 & 39 & 296 & 81 & 66 & 7,8 & \\
\hline 128 & 249 & 139 & 20 & 207 & 104 & 9 & 8 & $E$ & 155 & 372 & 300 & 20 & 310 & 80 & 67 & 3 & \\
\hline 14 & 108 & 187 & 6 & 204 & 103 & 10 & 4 & & 153 & 287 & 216 & 20 & 327 & 80 & 68 & 5,6 & \\
\hline 9 & 342 & 262 & 6 & 192 & 103 & 11 & 6 & $\mathrm{~K}$ & 219 & 83 & 283 & 32 & 274 & 80 & 69 & 1 & \\
\hline 54 & 374 & 318 & 19 & 200 & 98 & 12 & 3 & & 198 & 293 & 185 & 32 & 367 & 80 & 70 & 6 & \\
\hline 21 & 253 & 110 & 6 & 189 & 97 & 13 & 8 & 1 & 135 & 121 & 304 & 20 & 308 & 80 & 71 & 1 & \\
\hline 264 & 185 & 118 & 39 & 243 & 97 & 14 & 7,8 & D & 143 & 162 & 343 & 20 & 317 & 80 & 72 & 1 & \\
\hline 91 & 111 & 243 & 20 & 215 & 96 & 15 & 4 & & 249 & 172 & 319 & 36 & 294 & 80 & 73 & 1 & \\
\hline 15 & 285 & 86 & 6 & 209 & 96 & 16 & 9 & & 194 & 279 & 301 & 32 & 412 & 80 & 74 & 3 & \\
\hline 71 & 123 & 213 & 19 & 218 & 94 & 17 & 4 & & 130 & 228 & 95 & 20 & 302 & 79 & 75 & 8 & \\
\hline 189 & 328 & 328 & 32 & 229 & 94 & 18 & 3 & & 197 & 82 & 288 & 32 & 450 & 79 & 76 & 1 & \\
\hline 258 & 347 & 353 & 39 & 238 & 94 & 19 & 3 & & 243 & 318 & 251 & 36 & 369 & 79 & 77 & 6 & \\
\hline 110 & 230 & 92 & 20 & 239 & 92 & 20 & 8 & $\mathrm{~N}$ & 156 & 266 & 212 & 20 & 409 & 79 & 78 & 5,6 & \\
\hline 79 & 309 & 128 & 19 & 196 & 91 & 21 & 9 & G & 177 & 235 & 150 & 32 & 428 & 78 & 79 & 8 & \\
\hline 28 & 282 & 315 & 6 & 222 & 91 & 22 & 3 & & 201 & 300 & 314 & 32 & 514 & 78 & 80 & 3 & \\
\hline 104 & 105 & 187 & 20 & 279 & 90 & 23 & 4 & 0 & 257 & 257 & 146 & 39 & 338 & 78 & 81 & 8,9 & \\
\hline 206 & 284 & 143 & 32 & 304 & 88 & 24 & 8,9 & $F$ & 190 & 124 & 111 & 32 & 377 & 78 & 82 & 7 & \\
\hline 62 & 197 & 92 & 19 & 251 & 88 & 25 & 8 & & 238 & 309 & 280 & 36 & 315 & 78 & 83 & 3 & \\
\hline 119 & 377 & 201 & 20 & 257 & 88 & 26 & 6 & & 162 & 203 & 136 & 23 & 494 & 78 & 84 & 8 & \\
\hline 53 & 161 & 311 & 19 & 206 & 88 & 27 & 1 & & 228 & 206 & 146 & 32 & 343 & 78 & 85 & 8 & \\
\hline 56 & 219 & 90 & 19 & 253 & 87 & 28 & 8 & & 171 & 302 & 358 & 32 & 570 & 77 & 86 & 3 & \\
\hline 77 & 235 & 162 & 19 & 239 & 87 & 29 & 8 & & 172 & 106 & 187 & 32 & 570 & 77 & 87 & 4 & \\
\hline 105 & 277 & 342 & 20 & 234 & 87 & 30 & 3 & & 122 & 274 & 94 & 20 & 389 & 77 & 88 & 8,9 & \\
\hline 78 & 155 & 337 & 19 & 249 & 86 & 31 & 1 & $A$ & 192 & 323 & 290 & 32 & 369 & 77 & 89 & 3 & \\
\hline 87 & 323 & 257 & 20 & 282 & 86 & 32 & 6 & & 241 & 124 & 247 & 36 & 359 & 77 & 90 & 4 & \\
\hline 231 & 317 & 172 & 32 & 246 & 86 & 33 & 6,9 & & 252 & 325 & 319 & 36 & 376 & 77 & 91 & 3 & \\
\hline 211 & 270 & 311 & 32 & 208 & 86 & 34 & 3 & & 218 & 138 & 224 & 32 & 361 & 77 & 92 & 4 & \\
\hline 74 & 276 & 157 & 19 & 241 & 86 & 35 & 8,9 & & 209 & 300 & 147 & 32 & 425 & 77 & 93 & 9 & \\
\hline 89 & 205 & 118 & 20 & 257 & 86 & 36 & 8 & & 242 & 298 & 341 & 36 & 433 & 77 & 94 & 3 & \\
\hline 92 & 76 & 253 & 20 & 274 & 86 & 37 & 4 & & 41 & 235 & 144 & 15 & 428 & 77 & 95 & 8 & \\
\hline 158 & 118 & 85 & 21 & 256 & 86 & 38 & 7 & & 236 & 268 & 311 & 32 & 420 & 76 & 96 & 3 & \\
\hline 57 & 366 & 241 & 19 & 263 & 85 & 39 & 6 & & 227 & 330 & 286 & 32 & 523 & 76 & 97 & 3 & \\
\hline 90 & 133 & 335 & 20 & 264 & 85 & 40 & 1 & & 179 & 268 & 220 & 32 & 583 & 76 & 98 & 5,6 & \\
\hline 59 & 216 & 157 & 19 & 272 & 85 & 41 & 8 & & 256 & 277 & 302 & 39 & 488 & 76 & 99 & 3 & \\
\hline 106 & 344 & 252 & 20 & 257 & 85 & 42 & 6 & & 248 & 325 & 242 & 36 & 508 & 76 & 100 & 6 & \\
\hline 96 & 307 & 74 & 20 & 282 & 85 & 43 & 9 & & 181 & 134 & 232 & 32 & 443 & 76 & 101 & 4 & \\
\hline 98 & 115 & 242 & 20 & 239 & 84 & 44 & 4 & & 217 & 108 & 155 & 32 & 371 & 76 & 102 & 7 & \\
\hline 69 & 102 & 332 & 19 & 261 & 84 & 45 & 1 & & 245 & 221 & 161 & 36 & 398 & 76 & 103 & 8 & \\
\hline 114 & 275 & 125 & 20 & 290 & 84 & 46 & 8,9 & & 168 & 369 & 106 & 32 & 477 & 76 & 104 & 9 & \\
\hline 48 & 375 & 292 & 19 & 293 & 84 & 47 & 3 & & 175 & 226 & 137 & 32 & 250 & 75 & 105 & 8 & \\
\hline 163 & 296 & 320 & 32 & 305 & 84 & 48 & 3 & & 271 & 88 & 289 & 39 & 445 & 75 & 106 & 1 & \\
\hline 97 & 123 & 166 & 20 & 254 & 84 & 49 & 4,7 & & 140 & 304 & 116 & 20 & 438 & 75 & 107 & 9 & \\
\hline 210 & 321 & 339 & 32 & 218 & 84 & 50 & 3 & & 259 & 112 & 302 & 39 & 517 & 75 & 108 & 1 & \\
\hline 185 & 331 & 284 & 32 & 280 & 84 & 51 & 3 & & 246 & 97 & 265 & 36 & 323 & 75 & 109 & 4 & \\
\hline 180 & 97 & 285 & 32 & 248 & 83 & 52 & 1 & & 199 & 190 & 304 & 32 & 490 & 75 & 110 & 1 & \\
\hline 226 & 115 & 217 & 32 & 287 & 83 & 53 & 4 & & 205 & 195 & 113 & 32 & 484 & 75 & 111 & 8 & \\
\hline 125 & 291 & 319 & 20 & 265 & 83 & 54 & 3 & & 265 & 151 & 170 & 39 & 509 & 75 & 112 & 4,7 & \\
\hline 159 & 300 & 240 & 21 & 298 & 83 & 55 & 6 & & 267 & 371 & 122 & 39 & 329 & 75 & 113 & 9 & \\
\hline 160 & 372 & 118 & 21 & 294 & 82 & 56 & 9 & & 247 & 337 & 210 & 36 & 356 & 75 & 114 & 6 & \\
\hline 94 & 210 & 367 & 20 & 271 & 82 & 57 & 3 & & 174 & 291 & 152 & 32 & 375 & 74 & 115 & 9 & \\
\hline 165 & 352 & 334 & 32 & 274 & 82 & 58 & 3 & & 64 & 317 & 202 & 19 & 447 & 74 & 116 & 6 & \\
\hline
\end{tabular}




\section{LASER DAMAGE EXPERIMENTS}

As described in Ref. [1], the OSL Upgrade beam size is 10-cm x 13-cm elliptical. A 40-cm optic is accessed by the laser via different sub-apertures on the optic. The shot history of each OSL Upgrade sub-aperture for these experiments is shown in Table 2. 15 damage features (labeled A through O) were identified during a post-campaign inspection. Local fluence statistics at these 15 sites are given in Table 3. Microscope images of these 15 damage sites are shown in Figure 8. The key feature of Figure 8 is that all the damage sites identified map can be associated with lenslets located upstream of the damage (although not all lenslets are visible in the images). The distance $\mathrm{z}_{\mathrm{L}}$ from the output surface damage to the lenslet (corrected for index) is indicated in the images and in Table 3. Local fluence was obtained by taking the average fluence in a 5-mm patch at the damage site. Also note the damage at site $\mathrm{H}$ is in the bulk of the optic, and not at the output surface (as are all the others).

Table 2. Shot history of each of the sub-apertures (SA) shown in Figure 6.

\begin{tabular}{|c|c|c|c|}
\hline SA & $\begin{array}{c}\text { 3 } \omega \text { Pulse } \\
\text { width (ns) }\end{array}$ & $\begin{array}{c}\text { Pulse } \\
\text { shape }\end{array}$ & $\begin{array}{c}\text { \# of } \\
\text { shots }\end{array}$ \\
\hline 1 & 3.0 & Gaussian & 21 \\
\hline 3 & 3.0 & Gaussian & 11 \\
\hline 4 & 3.0 & Gaussian & 22 \\
\hline 6 & 3.0 & Gaussian & 11 \\
\hline 7 & 3.0 & Gaussian & 9 \\
\hline 8 & 2.0 & Square & 9 \\
\hline 9 & 2.0 & Square & 8 \\
\hline
\end{tabular}

Table 3. Statistics associated with the 15 lenslet-induced damage sites OSL campaign.

\begin{tabular}{|c|c|c|c|c||c|c|c|c|c|}
\hline Site & SA & $\begin{array}{c}\text { Ave. Local } \\
\text { Fluence }\end{array}$ & $\begin{array}{c}\text { Max. Local } \\
\text { Fluence }\end{array}$ & $\mathbf{Z}_{\mathbf{L}}(\mathbf{m m})$ & Site & SA & $\begin{array}{c}\text { Ave. } \\
\text { Local } \\
\text { Fluence }\end{array}$ & $\begin{array}{c}\text { Max. } \\
\text { Local } \\
\text { Fluence }\end{array}$ & $\mathbf{Z}_{\mathbf{L}}(\mathbf{m m})$ \\
\hline A & 1 & 4.7 & 6.9 & 22.7 & $\mathrm{G}$ & 9 & 4.4 & 5.1 & 13.7 \\
\hline B & 4 & 3.8 & 4.3 & 30.1 & $\mathrm{H}$ & 9 & 4.7 & 5.4 & 21.3 \\
\hline C & 7 & 3.8 & 5.1 & 30.3 & $\mathrm{I}$ & 8 & 4.5 & 5.1 & 36.0 \\
\hline C & 8 & 3.5 & 3.7 & - & $\mathrm{J}$ & 6 & 3.6 & 4.2 & 9.4 \\
\hline D & 7 & 3.8 & 5.5 & 36.5 & $\mathrm{~K}$ & 6 & 3.3 & 4.4 & 29.4 \\
\hline D & 8 & 3.2 & 3.4 & - & $\mathrm{L}$ & 2 & 2.1 & 2.2 & 6.0 \\
\hline E & 8 & 4.5 & 5.0 & 42.9 & $\mathrm{M}$ & 4 & 2.6 & 3.8 & 5.3 \\
\hline F & 8 & 3.8 & 4.4 & 41.6 & $\mathrm{~N}$ & 8 & 4.5 & 5.3 & 35.8 \\
\hline F & 9 & 4.5 & 5.3 & - & O & 4 & 4.1 & 5.5 & 19.8 \\
\hline
\end{tabular}



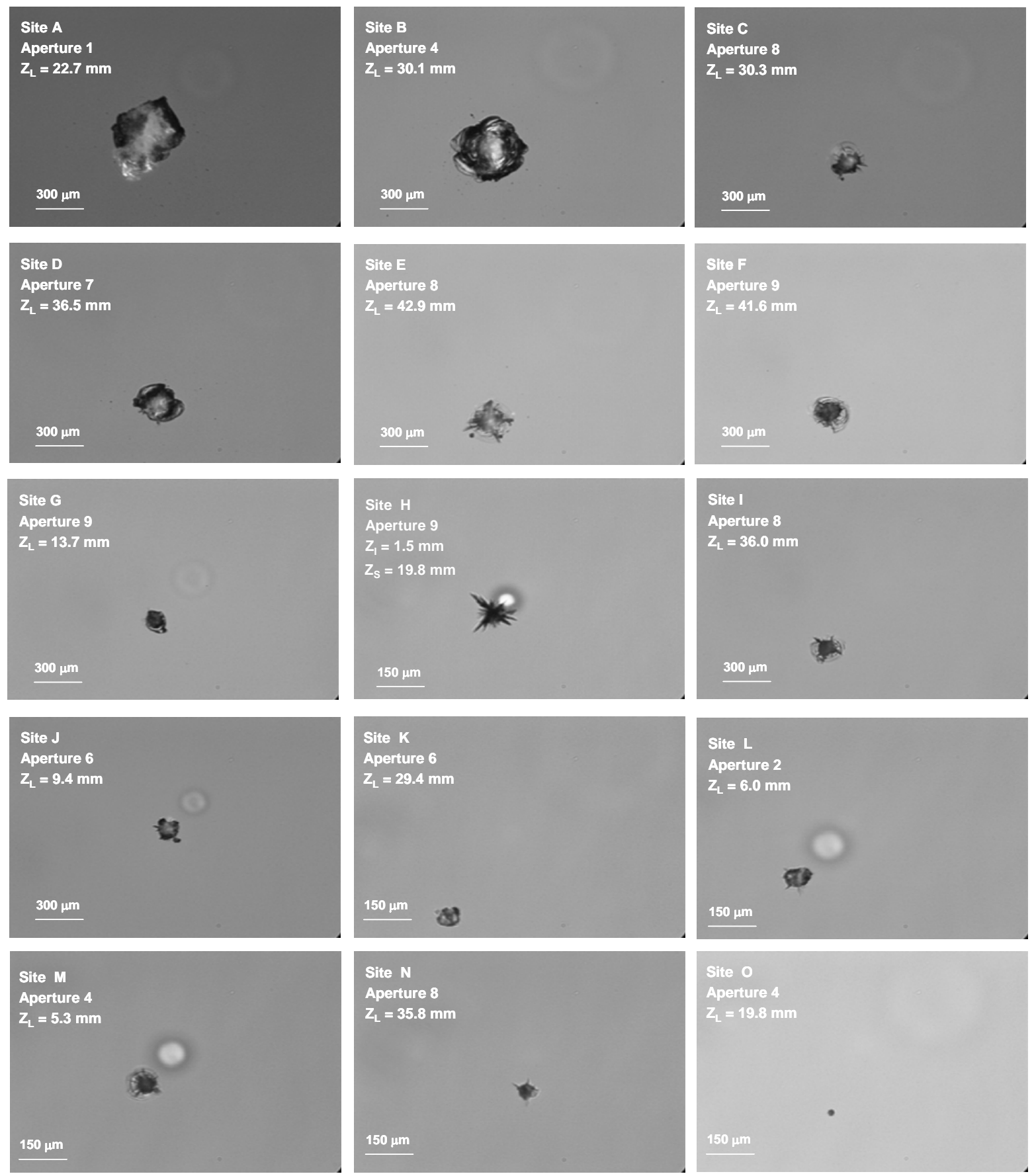

Figure 8. Microscope images of the 15 features identified in the post-campaign inspection. All 15 sites could be correlated to an upstream lenslet. The distance $\mathrm{z}_{\mathrm{L}}$ from the output surface to the lenslet is indicated along with the sub-aperture. Site $\mathrm{H}$ showed damage in the bulk, as thus also indicates the distance from the damage to the surface, $\mathrm{z}_{\mathrm{s}}$. 


\section{RESULTS AND ANALYSIS}

It is clear from Table 1 that a correlation exists between OPD and damage initiation, as the top five lenslets in terms of OPD all led to damage, and no lenslet below OPD rank of 31/116 led to damage. The larger lenslets that did not lead to damage were probably either: (a) at a "cold" spot in the beam, (b) at a z-location in the sample not favorable for large rear-surface intensification, or (c) were not actually lenslets (i.e. were surface features misidentified as lenslets). Likewise, the smaller lenslets that did lead to damage were probably (a) at a "hot" spot in the beam or (b) at a z-location that provided maximal intensification at the rear surface. In any case, this data indicates that no lenslet with an OPD of 85-nm or less led to damage at $4.5 \mathrm{~J} / \mathrm{cm}^{2}$, while all those with an OPD of 125 or greater led to damage at $4.5 \mathrm{~J} / \mathrm{cm}^{2}$.

It should be noted that the results of the phase-retrieval process are more difficult to interpret when the features are not pure lenslets (e.g. those that have associated opacities, and those that have multiple features). Nearly $25 \%$ of the 271 features fall in this category. This mostly affects the size determination, and has less of an effect on the OPD results. Some of the size data, therefore, will have large error bars associated with it. Also, the z-location determination is only accurate to with about $5 \mathrm{~mm}$, making it difficult to use this measurement as a filter for surface defects flagged by the pattern-recognition software.

Similar data was obtained from two other similar parts, omitted here for brevity. Figure 9 summarizes the statistics of the combined data. We find that no damage occurred from lenslets with an OPD less than $85 \mathrm{~nm}$, while damage always occurred from lenslets with an OPD more than $117 \mathrm{~nm}$. The phase-retrieval-derived OPD appears to be a good indicator of lenslet-induced damage potential.

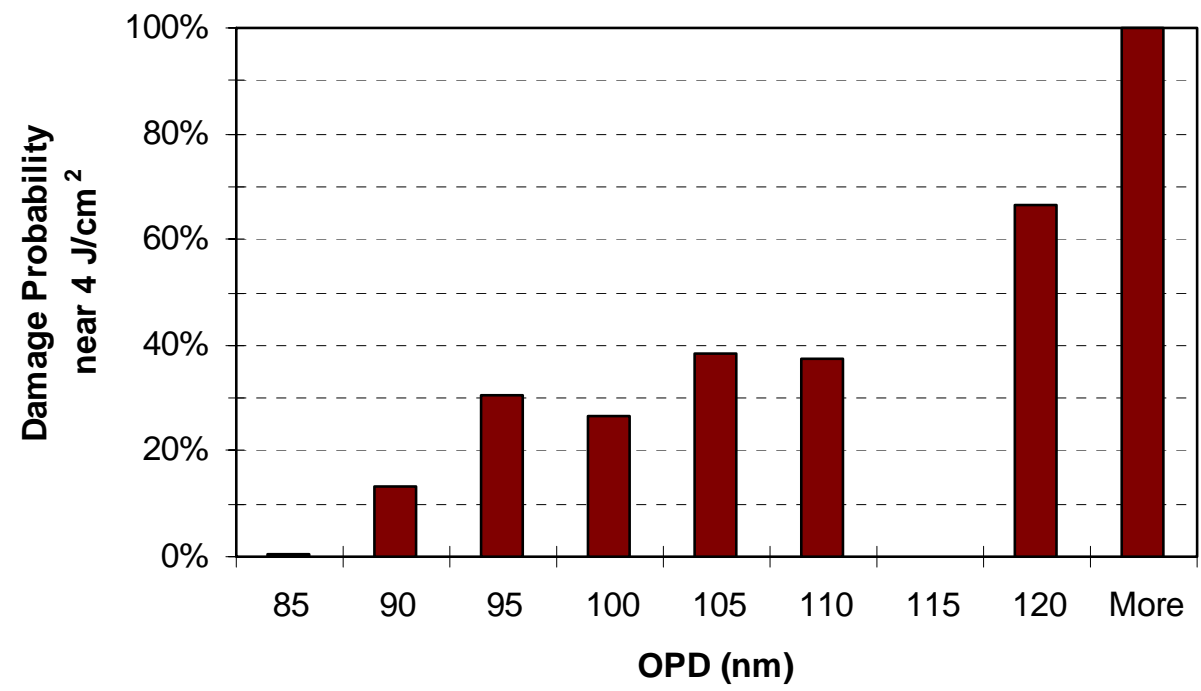

Figure 9. Lenslet-induced damage probability statistics from three Corning 7980 optics. All were shot near $4 \mathrm{~J} / \mathrm{cm}^{2}$. Only lenslets with an OPD of $70 \mathrm{~nm}$ or greater were detectable.

\section{GROWTH OF LENSLET-INDUCED DAMAGE}

Damage/lenslet sites D, E, F, G, H, I, N (which all were contained within a single OSL UPGRADE sub-aperture (SA 10)) were targeted for further laser shots in order to determine the nature of the lenslet-induced damage growth (i.e. mostly in $x-y$ ("lateral”), or in z (“drilling”)). The "drilling” hypothesis postulates that since light is being focused in the sample, a high-fluence core exists between the output-surface damage and the lenslet, resulting in a low-fluence ring away from the central core. The low-fluence ring would limit lateral growth, while the highfluence core would promote "drilling" back towards the lenslet, terminating when the fluence falls below a critical value. Shot statistics for this "growth" campaign are given in Table 4. 
Figure 10 shows the microscope images of the seven features targeted in the growth campaign. These images indicate major lateral growth can occur, and that "drilling" has not occurred at these sites. Generally speaking, the growth behavior of these sites is similar to sites studied previously which were not associated with lenslets ${ }^{3}$. Table 5 shows the local fluence statistics for each of the seven sites. A shot-by-shot fluence and ODI signal (Optical Damage Inspection system, see Ref [1]) history for the two largest sites (I and N) are shown in Figure 11. The ODI signal was obtained by summing pixel intensities at three different locations (of equal total pixel size): SIGNAL= location of the damage site; REF= location of a scatterer that did not grow, used to reference/normalize the input signal level; and BKG= location at edge of image to sample static background. The integrated signal (S) is then calculated by $\mathrm{S}=$ (SIGNAL-BKG)/(REF-BKG). In the figure, the ODI signal has been re-scaled to reflect the estimated diameter of the damage site by using the known sizes of the damage sites (before and after the growth campaign) for calibration and assuming a linear relationship between damage area and scatter signal. This assumption breaks down at larger diameters $(\sim 1.5-2 \mathrm{~mm})$ since only the high-frequency components scattering from the edge of the damage site contribute to the Schlieren signal (i.e. the scatter profile becomes donut-shaped). An exponential growth curve for site I is included in the figure, while site $\mathrm{N}$ does not appear to follow exponential growth.

Table 4. Shot statistics for the 42-shot growth campaign. Average and Maximum is taken over the number of shots. Fluence $\left(\mathrm{J} / \mathrm{cm}^{2}\right)$ and contrast are whole beam averages per shot.

\begin{tabular}{|c|c|c|c|c|}
\hline SA & $\begin{array}{c}\# \\
\text { Shots }\end{array}$ & $\begin{array}{l}\text { Ave. } \\
\text { Fluence }\end{array}$ & $\begin{array}{l}\text { Ave. } \\
\text { Contrast }\end{array}$ & $\begin{array}{l}\text { Max. } \\
\text { Fluence }\end{array}$ \\
\hline 10 & 42 & 4.0 & 0.176 & 4.9 \\
\hline
\end{tabular}
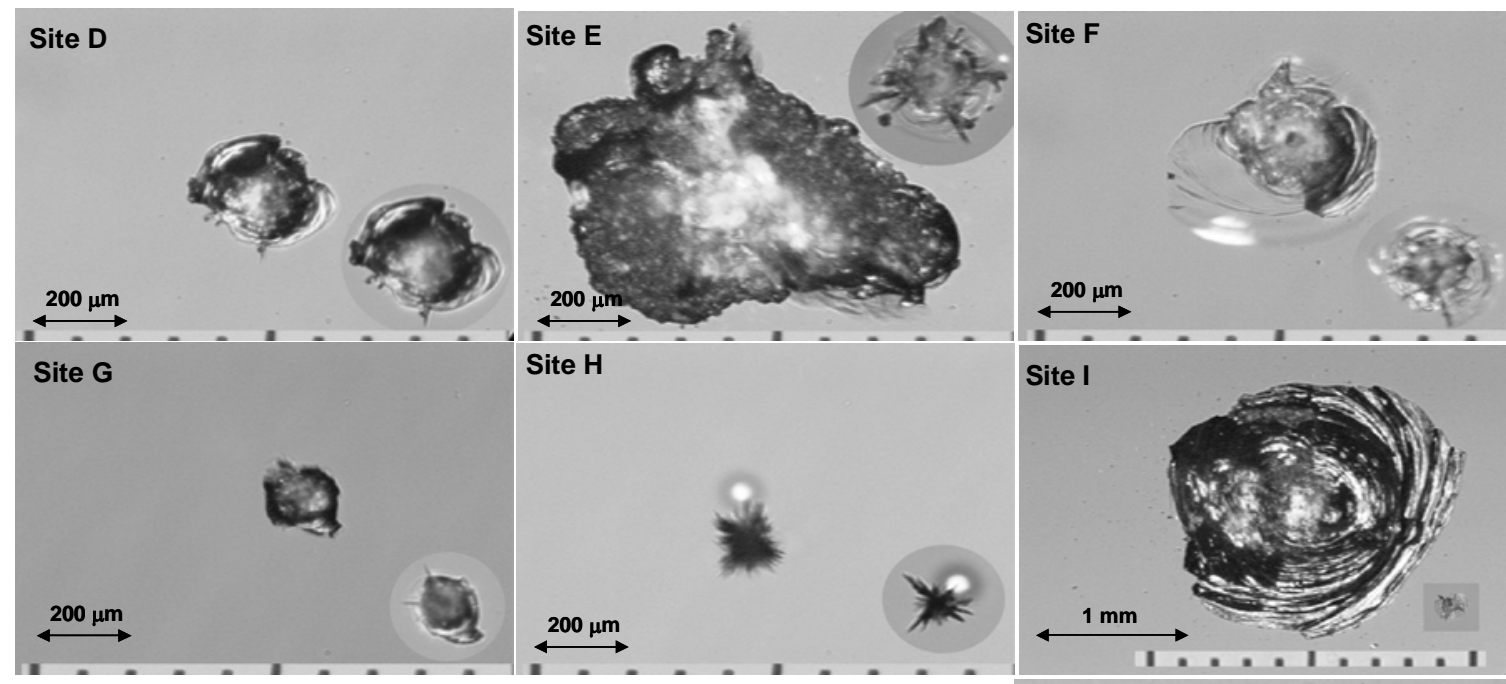

Figure 10. Microscope images of the seven damage sites targeted in the growth campaign. The insets show the damage after the initiation campaign (to scale) for comparison. Sites D and G showed little growth,

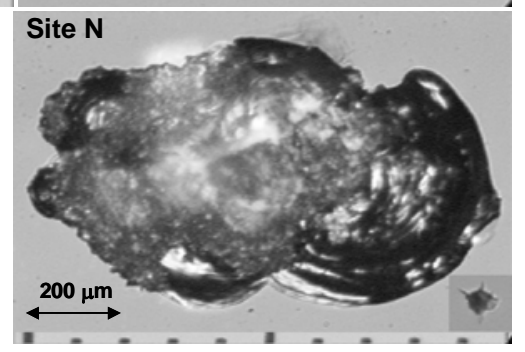
while I and N showed considerable growth. 
Table 5. Local fluences statistics for the seven lenslet sites targeted in 42-shot Round 2 campaign. Local fluence $\left(\mathrm{J} / \mathrm{cm}^{2}\right)$ refers to data averaged over a $\sim 5 \mathrm{~mm}$-diameter patch near the damage site.

\begin{tabular}{|l|l|l|l|l|l|l|l|}
\hline Lenslet Site & D & E & F & G & H & I & N \\
\hline Ave. Local Fluence & 3.5 & 5.0 & 4.7 & 3.9 & 4.5 & 4.6 & 3.8 \\
\hline St. Dev. Local Fluence & 0.5 & 0.6 & 0.6 & 0.6 & 0.6 & 0.5 & 0.6 \\
\hline Max. Local Fluence & 4.8 & 6.2 & 5.8 & 4.8 & 5.6 & 5.4 & 5.1 \\
\hline
\end{tabular}
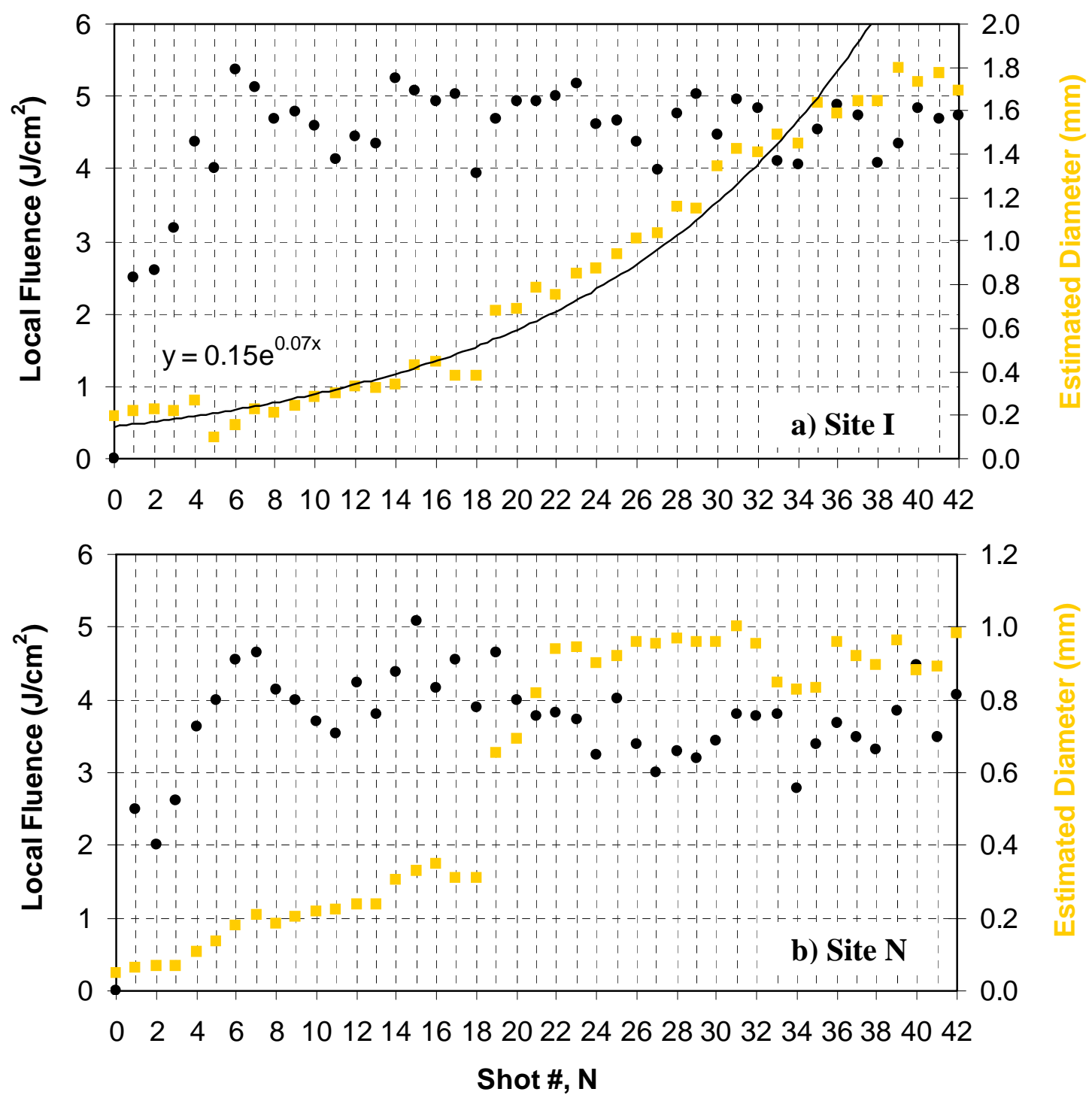

Figure 11. Estimated diameter and local fluence as a function of shot number for (a) lenslet/damage site I and (b) lenslet/damage site N. Diameter was estimated from the ODI signal intensity using the known diameter before shot \#1 and after shot \#42. Site I growth can be fit to an exponential (shown), while site $\mathrm{N}$ seems to have grown mostly in a few shots beginning at shot 19 . Recall that these 42 shots from the growth campaign followed $\sim 9$ shots from the initiation campaign, so that the total number of shots at each site was $\sim 50$. 


\section{CONCLUSIONS}

Experiments performed on the OSL Upgrade laser at $4 \mathrm{~J} / \mathrm{cm}^{2}$ (351-nm, 3-ns) have revealed a strong correlation between output surface damage and the optical path difference of phase defects (referred to as "lenslets") identified in the fused silica parts. Near $4 \mathrm{~J} / \mathrm{cm}^{2}$, lenslets with an OPD below $80-\mathrm{nm}$ were benign, while all of the lenslets with an OPD above 117-nm lead to damage. The growth of these sites upon further illumination at $4 \mathrm{~J} / \mathrm{cm}^{2}$ did not exhibit any unique characteristics. That is, the character of the growth rate and aspect ratio were comparable to our previous experience with output surface damage at sites not associated with lenslets; however, the measured growth rate was somewhat higher than expected from previous off-line measurements.

\section{ACKNOWLEDGMENTS}

A large amount of quality work on lenslets was performed by the late Bernie Penetrante of LLNL. We are indebted to his skilled efforts. This work was performed under the auspices of ther U.S. Department of Energy by the University of California, Lawrence Livermore National Laboratory under contract No. W-7405-Eng-48.

\section{REFERENCES}

${ }^{1}$ M.C. Nostrand, T.L. Weiland, R.L. Luthi, J.L. Vickers, W.D. Sell, J.A. Stanley, J. Honig, J. Auerbach, R.P. Hackel, and P.J. Wegner, "A large aperture, high energy laser system for optics and optical component testing," Laser-Induced Damage in Optical Materials 2003, G. J. Exarhos, A. H. Guenther, N. Kaiser, K.L. Lewis, M.J. Soileau, C.J. Stolz, Editors, Proceedings of SPIE Vol. 5273 (2004), p. 325.

${ }^{2}$ A.K. Burnham, L. Hackel, P. Wegner, T. Parham, L. Hrubesh, B. Penetrante, P. Whitman, S. Demos, J. Menapace, M. Runkel, M. Fluss, M. Feit, M. Key, T. Biesiada, "Improving 351-nm damage performance of largeaperture fused silica and DKDP optics,” Laser-Induced Damage in Optical Materials 2001, G. J. Exarhos, A. H. Guenther, K.L. Lewis, M.J. Soileau, C.J. Stolz, Editors, Proceedings of SPIE Vol. 4679 (2002), p. 173.

${ }^{3}$ M.A. Norton, L.W. Hrubesh, Z. Wu, E.E. Donohue, M.D. Feit, M.R. Kozlowski, D. Milam, K.P. Neeb, W.A. Molander, A. M. Rubenchik, W.D. Sell, P.J. Wegner, "Growth of laser-initiated damage in fused silica at 351 nm,” Laser-Induced Damage in Optical Materials 2000, G. J. Exarhos, A. H. Guenther, M. R. Kozlowski, K.L. Lewis, M.J. Soileau, Editors, Proceedings of SPIE Vol. 4347 (2001), p. 468. 